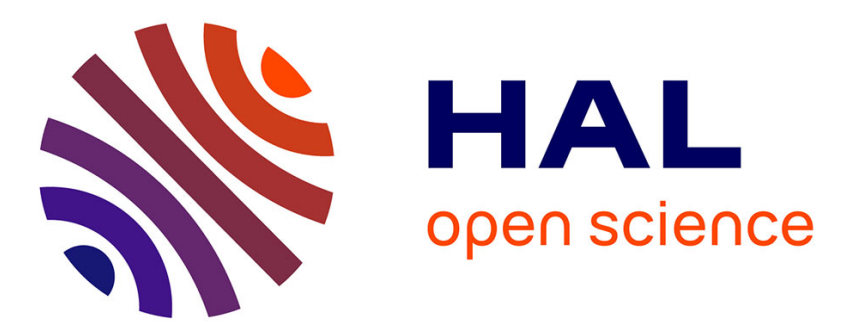

\title{
Cationic surfactants as a non-covalent linker for oxidised cellulose nanofibrils and starch-based hydrogels
}

Kazi Hossain, Vincenzo Calabrese, Marcelo da Silva, Saffron Bryant, Julien

Schmitt, Janet Scott, Karen Edler

\section{- To cite this version:}

Kazi Hossain, Vincenzo Calabrese, Marcelo da Silva, Saffron Bryant, Julien Schmitt, et al.. Cationic surfactants as a non-covalent linker for oxidised cellulose nanofibrils and starch-based hydrogels. Carbohydrate Polymers, 2020, 233, pp.115816. 10.1016/j.carbpol.2019.115816 . hal-03037406

\section{HAL Id: hal-03037406 https://hal.science/hal-03037406}

Submitted on 23 Nov 2021

HAL is a multi-disciplinary open access archive for the deposit and dissemination of scientific research documents, whether they are published or not. The documents may come from teaching and research institutions in France or abroad, or from public or private research centers.
L'archive ouverte pluridisciplinaire HAL, est destinée au dépôt et à la diffusion de documents scientifiques de niveau recherche, publiés ou non, émanant des établissements d'enseignement et de recherche français ou étrangers, des laboratoires publics ou privés. 


\title{
Cationic surfactants as a non-covalent linker for oxidised cellulose nanofibrils and starch-based hydrogels
}

\author{
Kazi M. Zakir Hossain ${ }^{\mathrm{a}}$, Vincenzo Calabrese ${ }^{\mathrm{a}}$, Marcelo A. da Silva ${ }^{\mathrm{a}}$, Saffron J. Bryant ${ }^{\mathrm{a}}$, \\ Julien Schmitt ${ }^{\mathrm{a}, \mathrm{1}}$, Janet L. Scott ${ }^{\mathrm{a}, \mathrm{b}}$, Karen J. Edler ${ }^{\mathrm{a}, \mathrm{b}, *}$ \\ ${ }^{a}$ Department of Chemistry, University of Bath, Claverton Down, Bath, BA2 7AY, United Kingdom \\ ${ }^{\mathrm{b}}$ Centre for Sustainable Chemical Technologies, University of Bath, Claverton Down, Bath, BA2 7AY, United Kingdom
}

A R T I C L E I N F O

\section{Keywords:}

Cellulose nanofibrils

Starch

Cationic surfactant

Rheology

\begin{abstract}
A B S T R A C T
Rheological properties of hydrogels composed of TEMPO-oxidised cellulose nanofibrils (OCNF)-starch in the presence of cationic surfactants were investigated. The cationic surfactants dodecyltrimethylammonium bromide (DTAB) and cetyltrimethylammonium bromide (CTAB) were used to trigger gelation of OCNF at around $5 \mathrm{mM}$ surfactant. As OCNF and DTAB/CTAB are oppositely charged, an electrostatic attraction is suggested to explain the gelation mechanism. OCNF ( $1 \mathrm{wt} \%)$ and soluble starch $(0.5$ and $1 \mathrm{wt} \%)$ were blended to prepare hydrogels, where the addition of starch to the OCNF resulted in a higher storage modulus. Starch polymers were suggested to form networks with cellulose nanofibrils. The stiffness and viscosity of OCNF-Starch hydrogels were enhanced further by the addition of cationic surfactants ( $5 \mathrm{mM}$ of DTAB/CTAB). $\zeta$-potential and amylose-iodine complex analyses were also conducted to confirm surface charge and interaction of OCNF-starch-surfactant in order to provide an in-depth understanding of the surfactant-induced gel networks.
\end{abstract}

\section{Introduction}

Cellulose is the most abundant polymer obtained from renewable biomass and has been widely used to form hydrogels. For example, cellulose nanofibrils can be obtained via the selective oxidation of the glucosyl C6 primary hydroxyl groups by $\mathrm{NaOCl}$ mediated with $(2,2,6,6-$ tetramethyl-piperidin-1-yl)oxyl (TEMPO)/NaBr, leading to the conversion of hydroxyl groups to carboxylate groups (Isogai, Saito, \& Fukuzumi, 2011; Saito, Kimura, Nishiyama, \& Isogai, 2007). This allows the formation of oxidised cellulose nanofibrils (OCNF) with a very large aspect ratio (length around a few hundreds nanometres and a cross-section below ten nanometres) (Nordenström, Fall, Nyström, \& Wågberg, 2017). The carboxylate groups provide the necessary electrostatic repulsion forces which are crucial to ensure a proper dispersion of the nanofibrils in aqueous solutions and the formation of hydrogels. OCNF hydrogels can be modulated by concentration (Geng et al., 2018), pH (Saito, Uematsu, Kimura, Enomae, \& Isogai, 2011), alcohols (da Silva et al., 2018), or in presence of additives, such as salt (Fukuzumi, Tanaka, Saito, \& Isogai, 2014), surfactants (Crawford et al., 2012) and block copolymers (Ingverud et al., 2016). For example,
OCNF hydrogels at different concentrations were investigated for their rheological properties and displayed promising shear-thinning properties for personal care applications (Crawford et al., 2012). Additionally, the high density of surface hydroxyl groups remaining on the OCNF surface even after the initial oxidation enables the nanofibrils to be further surface modified as required by specific applications (Azizi Samir, Alloin, \& Dufresne, 2005).

Alternatively, the surface carboxylate groups of OCNF can be employed to promote electrostatic interactions with cationic surfactants (Tardy et al., 2017). The charge reversal of anionic cellulose to a positive surface upon addition of a sufficient amount of cationic surfactant (as determined by $\zeta$-potential) has been reported in the literature (Prathapan, Thapa, Garnier, \& Tabor, 2016; Quennouz, Hashmi, Choi, Kim, \& Osuji, 2016). In addition, the improved rheological properties of cellulose nanofibril (Quennouz et al., 2016) and nanocrystal (Dhar, Au, Berry, \& Tam, 2012) hydrogels with the addition of a specific amount of cationic surfactant were investigated. For example, the addition of cationic surfactant (DTAB) below the CMC to cellulose nanofibril suspensions was key to obtain an increased gel modulus as well as retention of optical clarity (Quennouz et al., 2016).

\footnotetext{
* Corresponding author at: Department of Chemistry, University of Bath, Claverton Down, Bath, BA2 7AY, United Kingdom.

E-mail address: K.Edler@bath.ac.uk (K.J. Edler).

${ }^{1}$ Current address: LSFC - Laboratoire de Synthèse et Fonctionnalisation des Céramiques, UMR 3080 CNRS/Saint-Gobain CREE, Saint-Gobain Research Provence, 550 avenue Alphonse Jauffret, Cavaillon, France.
} 
Starch, the second most abundant polysaccharide after cellulose, is also widely used in the food (Saha \& Bhattacharya, 2010) and pharmaceutical (Hong, Liu, \& Gu, 2016) industries as an additive due to its excellent gelling and thickening properties. Starch mainly consists of amylose (which is predominantly linear glucose units) and amylopectin (branched glucose units). Amylose forms a left-handed helix with a hydrophilic surface and hydrophobic cavity (Immel \& Lichtenthaler, 2000; Putseys, Lamberts, \& Delcour, 2010), which favours the formation of inclusion complexes with hydrophobic moieties (Winter \& Sarko, 1974) impacting the key properties of starch, such as gelation, viscosity, and retrogradation (Putseys et al., 2010). Starch has been investigated with other polymers to form multicomponent hydrogels as an interpenetrating polymer network (Jin et al., 2013; Murthy, Mohan, Sreeramulu, \& Raju, 2006), which showed improved water diffusion and rheological properties (Gong, Katsuyama, Kurokawa, \& Osada, 2003; Haque, Kurokawa, \& Gong, 2012).

Both members of the polysaccharide family, starch and cellulose possess closely similar chemical structures made of glycosidic units, with the exception of their $\alpha$ - and $\beta$-linkage, respectively. Therefore, interactions between these two species arouse the interest of food, cosmetics and healthcare researchers. OCNF are strongly negatively charged ( $\zeta$-potential $\sim-55 \mathrm{mV}$ at $\mathrm{pH} \mathrm{7.0)}$ (Calabrese et al., 2018), while the $\zeta$-potential value for starch is also reported to be negative ( $\sim-19 \mathrm{mV}$ for native rice starch) (McNamee et al., 2018), therefore, the addition of counter-ions to the OCNF-starch blends can be used to screen electrostatic repulsion forces between OCNF and starch (Fukuzumi et al., 2014), hence improving the gel strength of OCNFstarch complexes by increasing their interactions. The stability of $\mathrm{OCNF} /$ water suspensions is often described as the balance between the non-covalent attractive interactions (such as van der Waals and hydrogen bonding) and electrostatic repulsive forces (Fall, Lindström, Sundman, Ödberg, \& Wågberg, 2011; Notley, 2008). Hence, understanding the aggregation mechanism of cellulose-surfactant-starch complexes in water is of crucial importance to control the hydrogel properties.

The aim of this study is to investigate the rheological properties of OCNF hydrogels in the presence of various concentrations of cationic surfactants (namely, DTAB and CTAB). OCNF (1 wt\%) and different concentrations of soluble starch $(0.5$ and $1 \mathrm{wt} \%)$ were also blended to form hydrogels before the addition of a fixed concentration $(5 \mathrm{mM})$ of cationic surfactant. In addition, the inclusion of surfactants within the amylose helices and formation of electrostatic linkages in the OCNFsurfactant-amylose system were investigated via amylose-iodine complex formation utilising a UV-vis spectrophotometer.

\section{Experimental}

\subsection{Materials}

OCNF ( ca 8 wt \% slurry in water) (da Silva et al., 2018) was produced using TEMPO/NaOCl/NaBr oxidation followed by high-pressure homogenisation of wood pulp (Saito et al., 2007). The degree of oxidation of OCNF was measured to be $\sim 25 \%$ by conductometric titration and reported previously (Courtenay et al., 2017; Johns et al., 2017). A never-dried OCNF stock solution $(2 \mathrm{wt} \%)$ was prepared after purification via dialysis under deionised (DI) water for 3 days (using cellulose acetate dialysis tubing MWCO 12400) and dispersed using a sonication probe ( $1 \mathrm{~s}$ on, $1 \mathrm{~s}$ off pulsed mode for a net time of $60 \mathrm{~min}$ at $30 \%$ amplitude, Ultrasonic Processor, FB-505). Ultra-pure DI water $(18.2 \mathrm{M} \Omega \mathrm{cm})$ was used for all dilutions and sample preparation.

Starch (soluble, S9765) was purchased from Sigma-Aldrich, UK. Amylose content in the starch was calculated to be $34 \%$ according to the method mentioned in (Sadasivam \& Manickam, 1996; Yuliana, Huynh, Ho, Truong, \& Ju, 2012) (details in the ESI 1). Also, according to the supplier (Sigma-Aldrich, UK), the soluble starch was processed from potatoes and the molecular weight of amylose fraction in potato starch has been reported to be in the range of 40,000-340,000 Da.

Starch stock solution $(2.5 \mathrm{wt} \%)$ was prepared by dissolving the required amount of starch in DI water at $80^{\circ} \mathrm{C}$ for $45 \mathrm{~min}$ under continuous stirring. Gels were prepared by adding the hot starch solution $\left(80^{\circ} \mathrm{C}\right)$ into the OCNF suspension $\left(25^{\circ} \mathrm{C}\right)$ at various ratios of OCNF and starch (1:0.5 and 1:1 wt\%) followed by immediate vortex mixing while still warm, after mixing the suspension was allowed to cool to room temperature $\left(25^{\circ} \mathrm{C}\right)$ for gelation. The required amount of cationic surfactants (Dodecyltrimethylammonium bromide (DTAB, purity $\geq 98 \%$, MW $308.34 \mathrm{~g} \mathrm{~mol}^{-1}$, Sigma-Aldrich, UK), cetyltrimethylammonium bromide (CTAB, purity $99 \%$, MW $364.45 \mathrm{~g} \mathrm{~mol}^{-1}$, ACROS Organic, Fisher-Scientific, UK) were added to the starch solution prior to mixing with OCNF during the gel formation. All hydrogels were stored at $4{ }^{\circ} \mathrm{C}$ until characterisation.

\subsection{Characterisation}

\subsubsection{Electron microscopic analysis}

The morphology of the OCNF was examined using transmission electron microscopy (TEM) on a JEOL (JEM-2100 Plus, USA) at an operating voltage of $200 \mathrm{kV}$. The Cu-grid (mesh size 300 ) containing the sample $(0.025 \mathrm{wt} \%)$ was stained negatively using uranyl acetate (from Sigma-Aldrich, UK) (2 wt\%) for $1 \mathrm{~min}$ for enhanced contrast.

\subsubsection{Surface charge and particle size measurement}

$\zeta$-potential measurements were conducted using a Zeta-sizer (Malvern Zeta-sizer Nano ZSP ${ }^{\oplus}$, UK). Dilute solution $(0.1 \mathrm{wt} \%)$ of samples in DI water were placed in the folded capillary electrode cell and the $\zeta$-potentials measured as an average of 5 measurements from 100 scans each.

\subsubsection{Rheological analysis}

Rheological tests were performed using a stress-controlled rheometer (Discovery HR-3, TA Instruments, USA) equipped with a sandblasted plate-plate stainless steel geometry $(40 \mathrm{~mm})$ at $25^{\circ} \mathrm{C}$. Approximately $1 \mathrm{~mL}$ of gel was placed between the plates (with a plateplate gap of $0.5 \mathrm{~mm}$ ) and frequency, amplitude and flow sweeps were measured in order to determine the viscoelastic properties of the gels. Frequency sweeps were conducted, within the linear viscoelastic range, in strain control mode at $0.5 \%$ strain with an angular frequency range from $0.1-100 \mathrm{rad} \mathrm{s}^{-1}$. Amplitude sweeps were measured at an angular frequency of $1 \mathrm{~Hz}\left(6.28 \mathrm{rad} \mathrm{s}^{-1}\right)$ covering the strain ranging from $0.01-50 \%$. Finally, flow curves were measured to study the viscosity response of the sample to shearing, with a shear rate ranging from $0.01-100 \mathrm{~s}^{-1}$.

\subsubsection{UV-vis spectrophotometry}

Iodine stock solution (50\% of Lugol's solution) was prepared by dissolving $0.25 \mathrm{~g}$ of iodine (Fisher Scientific, UK) and $0.5 \mathrm{~g}$ of potassium iodide (Sigma-Aldrich, UK) in $150 \mathrm{~mL}$ of DI water under magnetic stirring. Then $30 \mu \mathrm{l}$ of the prepared iodine solution was added to each $5 \mathrm{~mL}$ of diluted (25 times) gel sample before measuring the absorbance using a UV/visible spectrometer (Varian Cary 50 Probe) by scanning over the wavelength range of $290-800 \mathrm{~nm}$.

\section{Results and discussion}

The demand for utilisation of bio-based materials in various formulation-based products is increasing rapidly to ensure a sustainable future. Here we investigated cellulose nanofibrils and starch, both derived from renewable sources, in fabricating shear-thinning hydrogels with the aid of cationic surfactants that have the potential to be utilised as an alternative to traditional synthetic rheology modifiers.

The morphology of never-dried OCNF was characterised using TEM revealing long fibrils with a length of hundreds of $\mathrm{nm}$ and a crosssection diameter around $5 \mathrm{~nm}$, as can be seen in Fig. 1. The diameter 


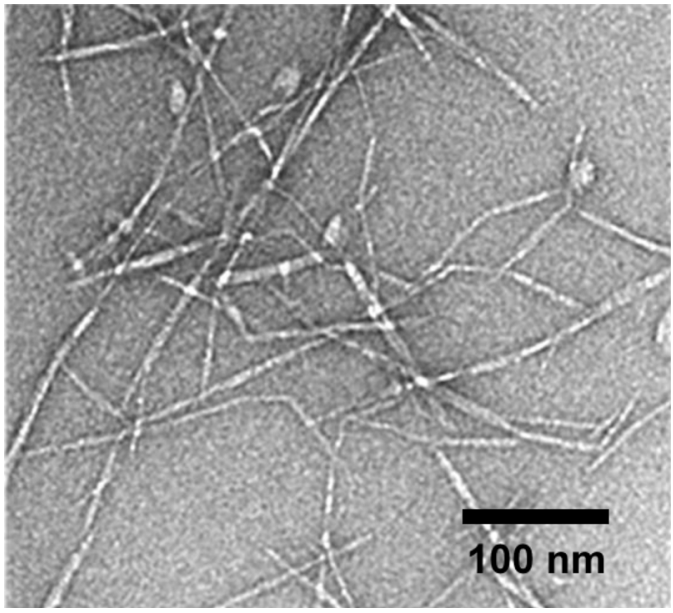

Fig. 1. TEM image of never-dried OCNF.

and the length of the fibrils were reported earlier to be $D=7 \pm 2 \mathrm{~nm}$ and $L=160 \pm 60 \mathrm{~nm}$, respectively (from averaging 175 measurements) (Schmitt et al., 2018).

Addition of cationic surfactants (DTAB and CTAB) at various concentrations $(1,5,10 \mathrm{mM})$ in the aqueous dispersion of OCNF (1 wt\%) were studied for gelation properties and their representative images are presented in Fig. 2a. Both DTAB and CTAB at low concentration (i.e., $1 \mathrm{mM}$ ) with OCNF (1 wt\%) were not observed to form a self-standing gel as indicated in Fig. 2a-ii\&v. On the other hand, stronger selfstanding gels were observed to form upon addition of $5 \mathrm{mM}$ of surfactants (Fig. 2a-iii\&vi). At higher concentrations $(10 \mathrm{mM})$ of cationic surfactants with OCNF ( $1 \mathrm{wt} \%$ ), the stability of the gels was severely reduced, as the suspensions were seen to flow (Fig. 2a-iv\&vii) and syneresis was observed after $24 \mathrm{~h}$ of gel formation. The larger amount of cationic surfactant induced a significant amount of fibril aggregation leading to loss of optical clarity, and to phase separation (Quennouz

a) DTAB CTAB

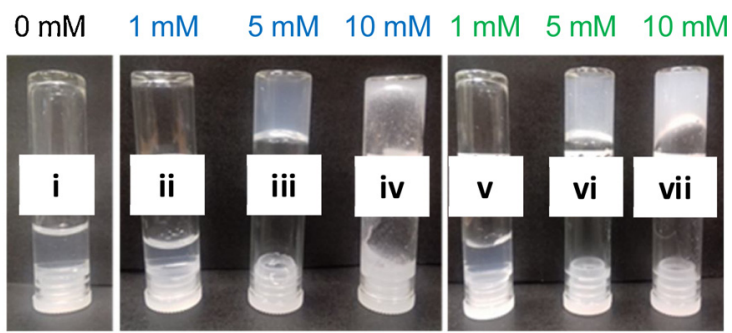

b)

Surfactant concentration (mM)

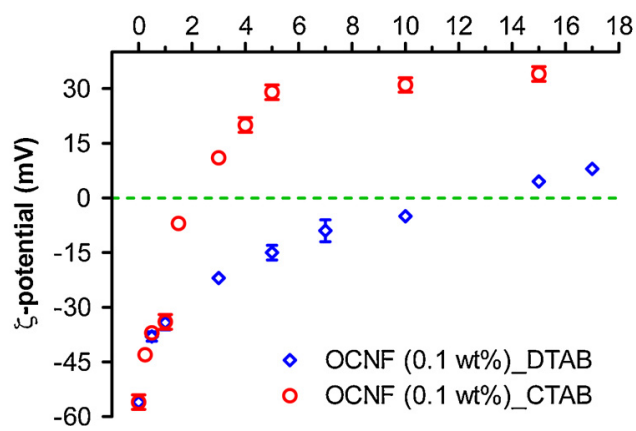

Fig. 2. a) Photographs of OCNF ( $1 \mathrm{wt} \%$ )/surfactant hydrogels produced with various concentrations of cationic surfactants : (i) Control $(0 \mathrm{mM})$, (ii) $1 \mathrm{mM}$, (iii) $5 \mathrm{mM}$, (iv) $10 \mathrm{mM}$ of DTAB, (v) $1 \mathrm{mM}$, (vi) $5 \mathrm{mM}$ and (vii) $10 \mathrm{mM}$ of CTAB, and b) $\zeta$-potential values of diluted OCNF $(0.1 \mathrm{wt} \%) /$ surfactants systems. et al., 2016).

The stability of the OCNF/surfactants gels can also be rationalised by considering the electrostatic interactions between the cationic surfactant headgroups and the anionic cellulose nanofibrils, as can be indicated by their $\zeta$-potential values, which was reported to be partially neutralised by the addition of cationic surfactant (Prathapan et al., 2016; Quennouz et al., 2016). Fig. 2b shows the $\zeta$-potential values of the OCNF $(0.1 \mathrm{wt} \%)$ suspensions at various surfactant concentrations. The $\zeta$-potential value of OCNF alone was $-56( \pm 2) \mathrm{mV}$ and with the addition of DTAB and СTAB the $\zeta$-potential shifted toward positive values, indicating neutralisation of the OCNF charge by cationic surfactant addition. The $\zeta$-potential values of both OCNF $(0.1 \mathrm{wt} \%) / \mathrm{DTAB}$ and OCNF $(0.1 \mathrm{wt} \%) / \mathrm{CTAB}$ systems remained negative at surfactant concentration below their CMC (the CMC value of CTAB is $\sim 1.1 \mathrm{mM}$ and DTAB is $\sim 14.0 \mathrm{mM}$ (Moulik, Haque, Jana, \& Das, 1996; Tedeschi et al., 2003)). However, just above the CMC both surfactants displayed charge inversion towards positive values suggesting the complete coverage of anionic cellulose nanofibrils with the cationic surfactant micelles. Similar charge neutralisation as well as charge inversion phenomenon in cellulose nanocrystal/cationic surfactants systems has been reported by Brinatti et al. (Brinatti, Huang, Berry, Tam, \& Loh, 2016).

The storage modulus $\left(G^{\prime}\right)$ of OCNF/surfactant gel systems were seen to increase with the cationic surfactant content, as illustrated in Fig. 3a-b. The control OCNF (1 wt\%) and the gels formed with a lower quantity of surfactant ( $1 \mathrm{mM} \mathrm{DTAB} / \mathrm{CTAB})$ showed a frequency dependant linear increase of $\mathrm{G}^{\prime}$, which demonstrated the dynamic nature of the transient interactions among the fibrils (Kavanagh \& RossMurphy, 1998). However, at higher concentration of surfactant (5 mM and above), G' for both the OCNF/DTAB and OCNF/CTAB gels were found to be higher, as well as less frequency dependent (represented by the negligible slope of the curves in Fig. 3a-b) suggesting the formation of more stable static interactions among the fibrils in the presence of oppositely-charged surfactants. The increase of G' (and with it of the gel stiffness) with the surfactant concentration ( $\tan \delta$ values in ESI 2), coupled with a less-charged surface as measured by $\zeta$-potential measurements, suggest that electrostatic attraction between OCNF and $\mathrm{DTAB} / \mathrm{CTAB}$ headgroup due to their opposite charges leads to neutralisation of repulsive forces and so to the formation of a connected network between the fibrils.

However, the effect of a wider range of surfactant concentrations on the rheological properties cannot be explored. As shown in the photo image in Fig. 2a, a mixture of $1 \mathrm{wt} \%$ OCNF with $10 \mathrm{mM} \mathrm{DTAB}$ had strong phase seperation. This syneresis would have been exacerbated by further increases in the DTAB concentration. Therefore, higher concentrations of DTAB, including above the CMC $(14 \mathrm{mM})$ were not explored. On the other hand, the concentration range included values above and below the CMC of CTAB. As the lowest CTAB concentration $(1 \mathrm{mM})$ did not result in self-standing gel formation, then even lower concentrations were not explored.

From the amplitude sweep curves (see Fig. 3c) the linear viscoelastic (LVE) region of the OCNF/DTAB gel systems were found to be extended to a strain of $10.0 \%$ (5 mM DTAB) and $1.5 \%$ (10 mM DTAB), beyond which the quiescent gel structure was lost. The highest DTAB content (10 $\mathrm{mM}$ ) within the OCNF gels was found to cause phase separation due to syneresis, which reduces the LVE region compared to $5 \mathrm{mM}$ (as indicated in Fig. 3c). On the other hand, the OCNF/CTAB gels showed their LVE region extended to a strain of $4.0 \%$ (for both 5 and $10 \mathrm{mM}$ CTAB content gels) as presented in Fig. 3d. At $5 \mathrm{mM}$ concentration, the OCNF/CTAB gels showed a lower LVE region compared to the OCNF/ DTAB gels, this could be due to the effect of strain on the CTAB micelles (CMC value of CTAB $\sim 1.1 \mathrm{mM}$ and DTAB $\sim 14.0 \mathrm{mM}$ (Moulik et al., 1996; Tedeschi et al., 2003)) present in the gel systems. This correlates with the optical clarity of the gels obtained (Fig. 2a), where more cloudy gels were seen in case of OCNF/CTAB compared to the OCNF/ $\mathrm{DTAB}$ at $5 \mathrm{mM}$ concentration. However, at $10 \mathrm{mM}$ concentration, OCNF/DTAB gels showed a lower LVE region compared to the OCNF/ 

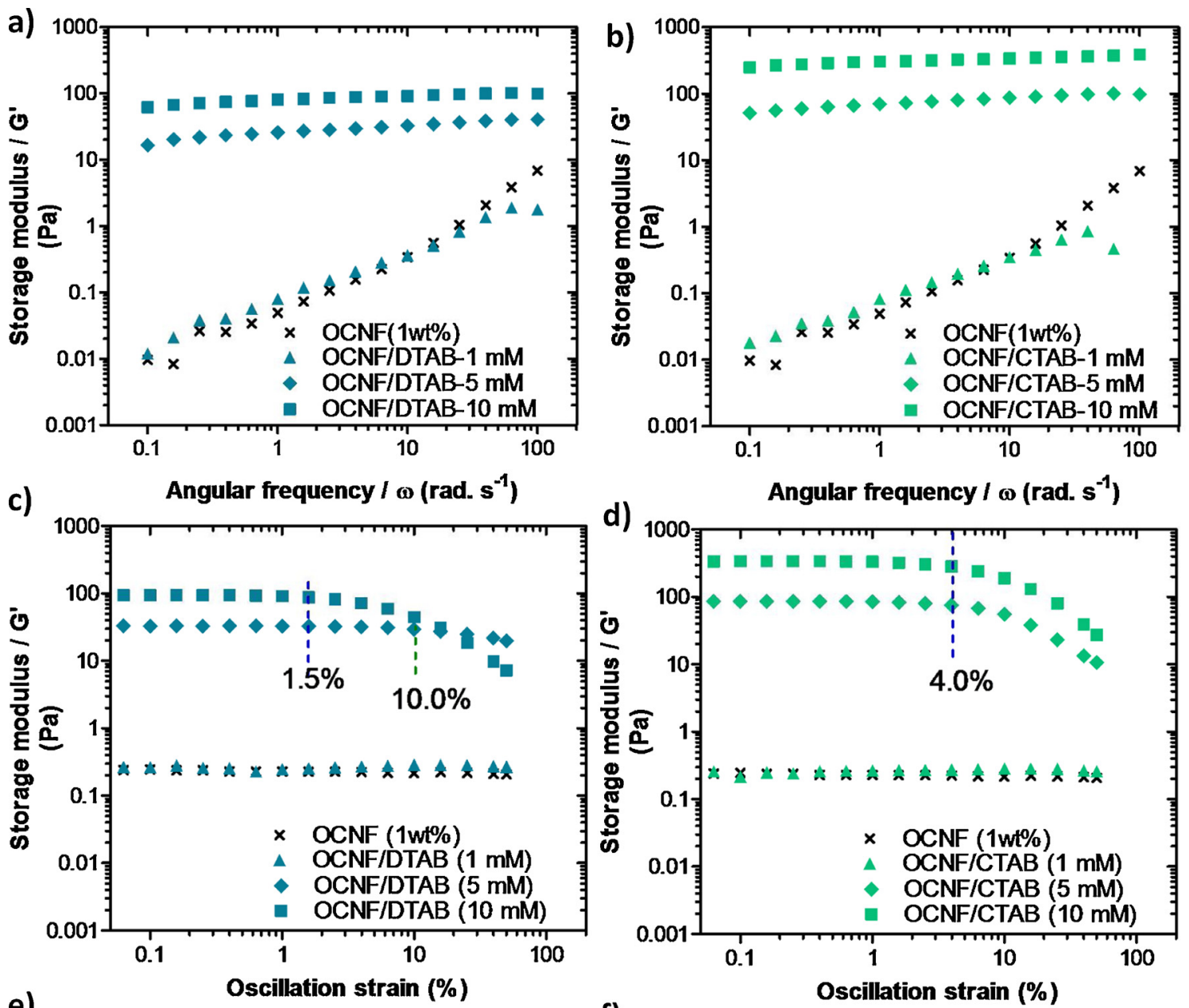

e)
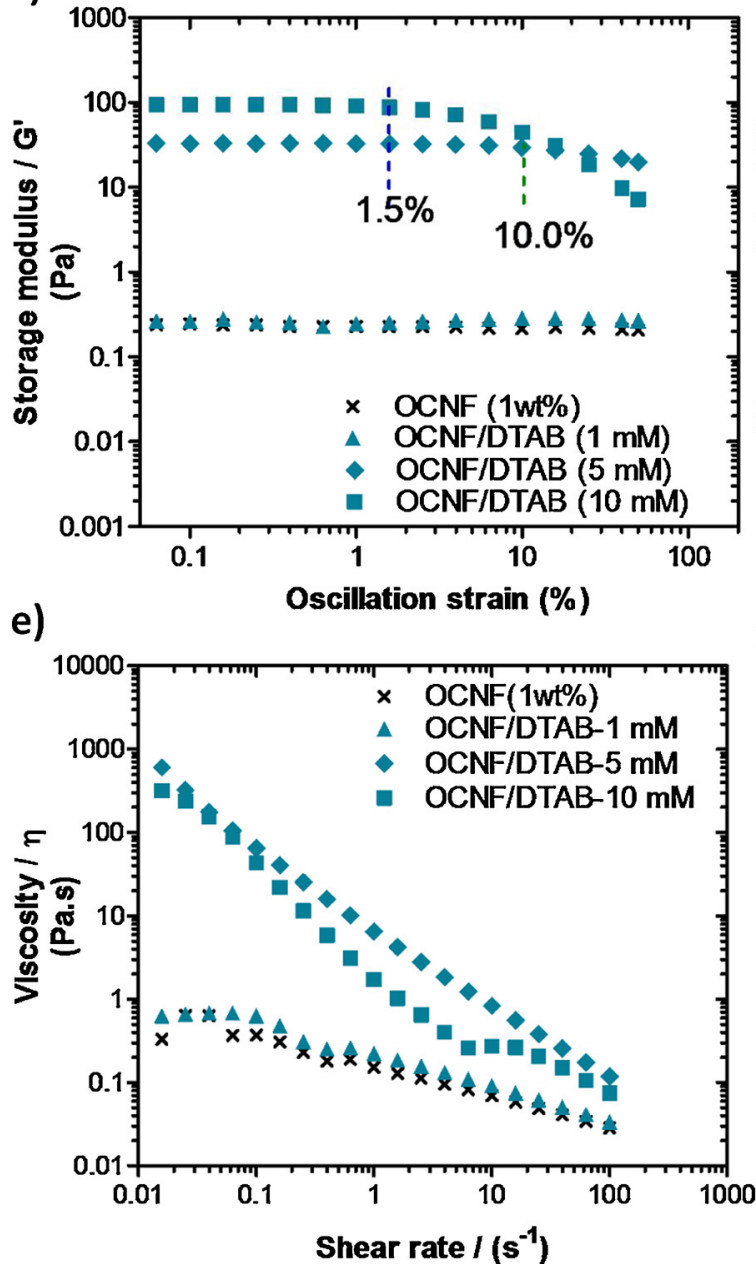

f)

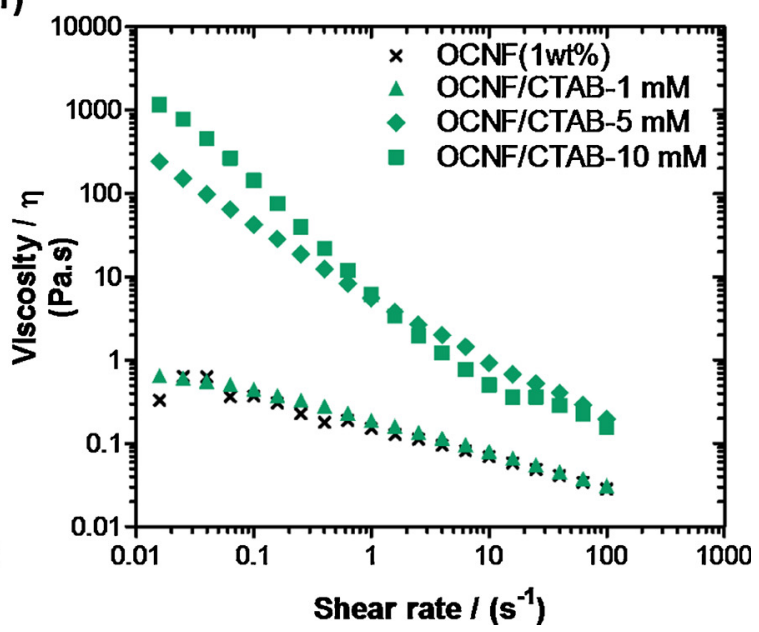

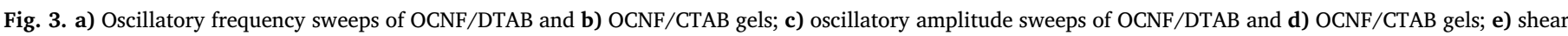
flow curves of OCNF/DTAB and f) OCNF/CTAB gels.

$\mathrm{CTAB}$ gels due to the greater syneresis observed for that gel system, as shown earlier in Fig. $2 \mathrm{a}$.

The flow behaviour of the gels was also investigated by disrupting the network under a constant increase in shear rate. The dependence of apparent viscosity $(\eta)$ with respect to the shear rate of the gel systems are presented in Fig. 3e-f. The control OCNF (1 wt\%) and the gels produced using various concentrations of cationic surfactants (DTAB/ $\mathrm{CTAB}$ ) were observed to possess shear-thinning properties. The addition of $1 \mathrm{mM}$ surfactant content in the OCNF gels did not cause any significant change in their $\eta$ values and shear dependence. For higher surfactant content OCNF gels (5 and $10 \mathrm{mM}$ DTAB/CTAB), a strong effect was observed marked by a significant increase of $\eta$ and shear dependency. Even at high shear rate, $\eta$ diverges strongly from OCNF samples at lower surfactant concentrations, which suggests the presence of larger aggregates.

Starch can also form shear-thinning gels on its own at high weight fraction (above 4-6 wt\%, depending on the source) (Miles, Morris, Orford, \& Ring, 1985; Morris, 1990).

Gels made by mixtures of OCNF and starch were studied with a fixed weight fraction of cellulose ( $1 \mathrm{wt} \%$ of OCNF) and different weight 
a)
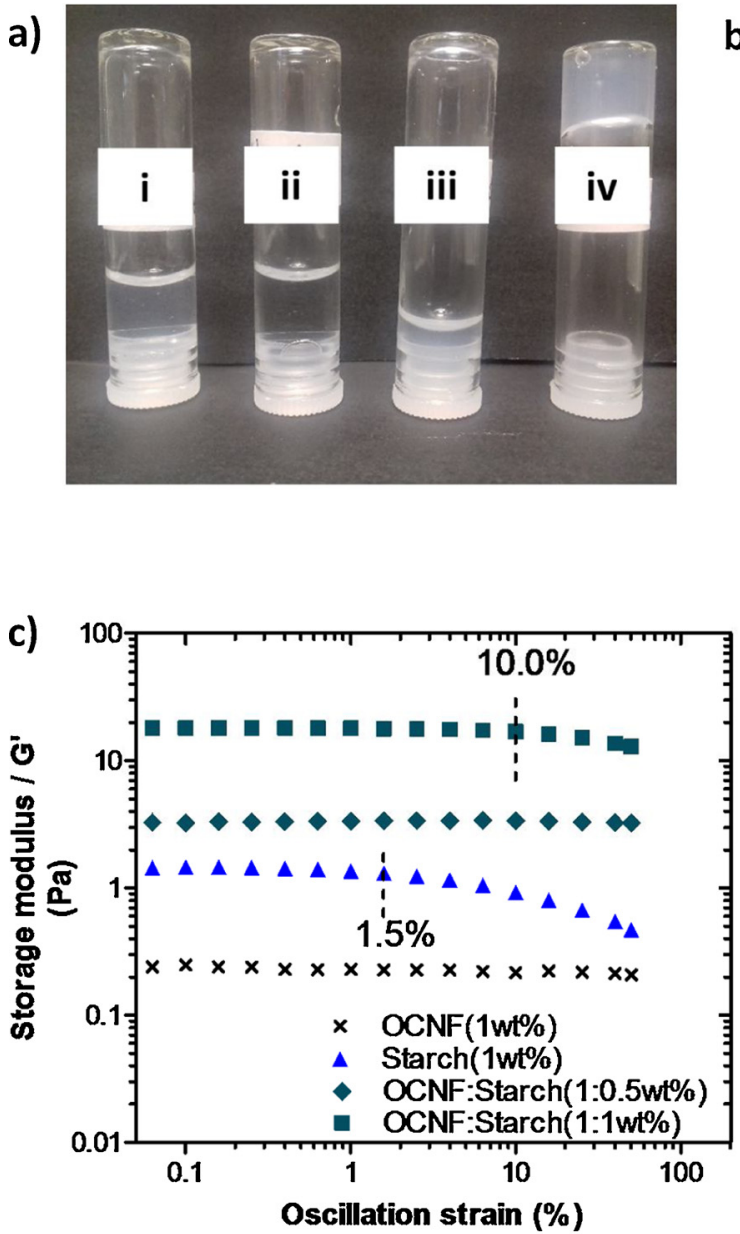

b)

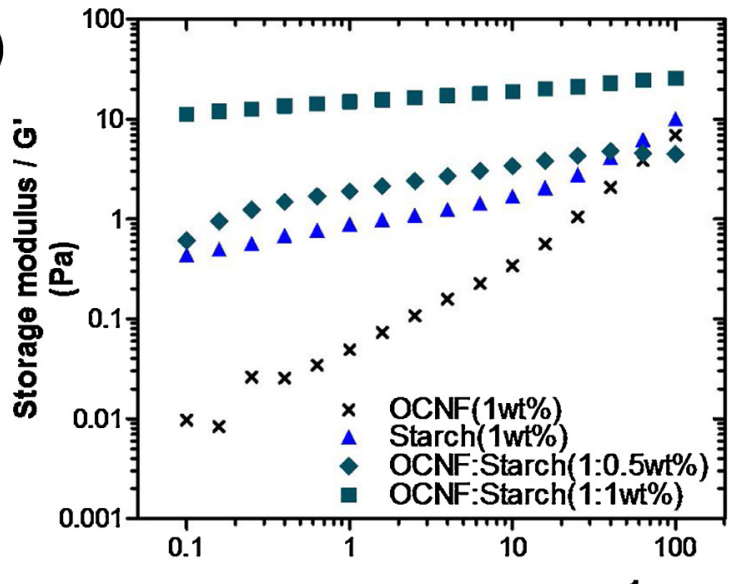

d)

Angular frequency $I \omega\left(\right.$ rad. $\left.\mathrm{s}^{-1}\right)$

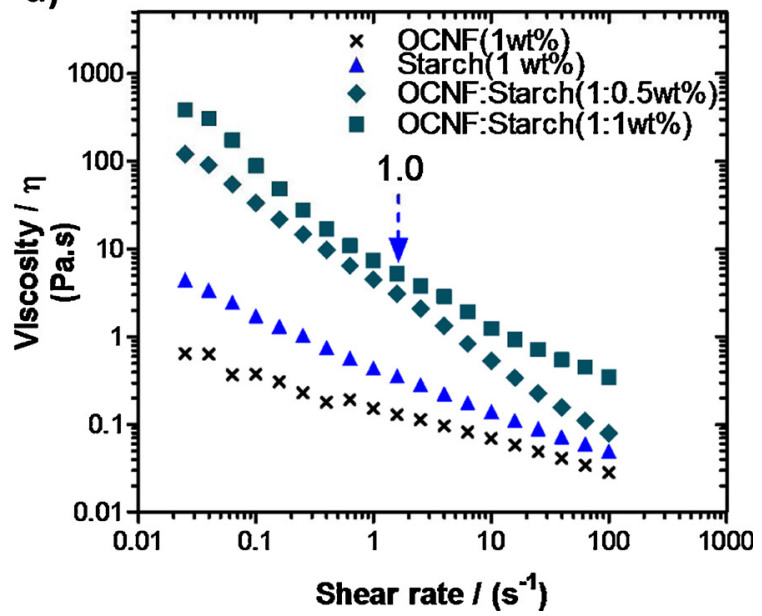

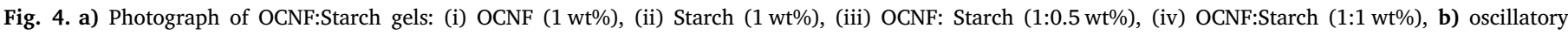
frequency sweeps, c) oscillatory amplitude sweeps and d) shear flow curves of OCNF:Starch gels.

fractions of starch $(0.5,1 \mathrm{wt} \%$ of soluble starch, labelled as Starch). Both OCNF (1 wt\%) and Starch (1 wt\%) were observed to be liquid-like and optically clear as can be seen in Fig. 4a, which demonstrated their homogeneous dispersion in DI water. However, the addition of starch ( 0.5 and $1 \mathrm{wt} \%$ ) to the OCNF ( $1 \mathrm{wt} \%$ ) led to an increase in opacity suggesting the formation of aggregates and leading to comparatively stiffer gels (self-standing) for the OCNF:Starch (1:1 wt\%) mixture (Fig. 4a). The OCNF:Starch gels were stable (no precipitaion was seen after $48 \mathrm{~h}$, during the rheology test). Addition of $1 \mathrm{wt} \%$ starch to $1 \mathrm{wt} \%$ OCNF caused a slight reduction in $\zeta$-potential to $-48 \pm 1 \mathrm{mV}$, but it was still above the established minimum value of $-30 \mathrm{mV}$ required for dispersion stability (Han, Zhou, Wu, Liu, \& Wu, 2013).

Fig. 4c-e compare the rheological properties of OCNF:Starch hydrogels. The control Starch (1 wt\%) gels showed a weaker dependence of frequency, suggesting a solid-like behaviour ( $G^{\prime}>$ G", see ESI 3) compared to the control OCNF (1 wt\%) ('GG" > G', see ESI 3). However, the OCNF:Starch blends at different starch $\mathrm{wt} \%$ revealed a strong increase of G' compared to the control OCNF (1 wt\%) and Starch (1 wt $\%)$ gels, as can be seen in Fig. 4c. For example, OCNF:Starch (1:1 wt\%) gels had almost 15 times higher values of $G^{\prime}$ (with very low-frequency dependency and more pronounced G' $>$ G'/G' ratio, see ESI 3) compared to the control Starch ( $1 \mathrm{wt} \%)$ gels. This might be due to the presence of higher weight fraction of solids as well as their contribution towards the formation of a denser network. However, starch gels containing similar solid content (i.e. $2 \mathrm{wt} \%$ ) revealed significantly lower values of $\mathrm{G}^{\prime}$ compared to the OCNF:Starch (1:1 wt\%) gels (see ESI 4). Although, the pure OCNF ( $2 \mathrm{wt} \%$ ) showed slightly higher G' compared to the OCNF:Starch (1:1 wt\%) gels (ESI 4), this is expected as the OCNF is the main building block for stiffer gel formation due to their fibrillar structure (higher aspect ratio) as well as their higher negative surface charge.

From the amplitude sweep curves (see Fig. 4d), the LVE region for Starch ( $1 \mathrm{wt} \%)$ was found to be extended to a strain of $1.5 \%$, while the stiffer OCNF:Starch (1:1 wt \%) gels showed the LVE region up to $10.0 \%$ strain. However, OCNF:Starch (1:0.5 wt\%) gels showed the LVE region up to $50 \%$ strain suggesting the presence of a less aggregated network (i.e., more well-dispersed solids). Both of the OCNF:Starch blends showed higher shear $\eta$ with increasing starch content compared to the control OCNF and Starch gels; however, all of them possess shearthinning properties, as can be seen in Fig. 4e.

As $5 \mathrm{mM}$ of both surfactants (DTAB/CTAB) was found to trigger gelation in OCNF/surfactant gels (Fig. 2), the concentration of surfactant was kept at this value in the OCNF/Starch/surfactant gel systems investigated here. Addition of $5 \mathrm{mM}$ DTAB to the OCNF:Starch blends showed further enhancement of the storage modulus compared to OCNF/DTAB gels (see Fig. 5a) suggesting the formation of a noncovalent link mediated by the surfactant between the OCNF and starch networks. This effect is greater in the higher solid fraction OCNF:Starch (1:1 wt $\%)$ gels than for the OCNF:Starch (1:0.5 wt\%) gels. In contrast, while both OCNF:Starch gels also showed an enhancement of the storage modulus after addition of $5 \mathrm{mM} \mathrm{CTAB}$, the higher solid fraction OCNF:Starch (1:1 wt\%) gels did not show any noticeable difference compared to the lower solid fraction of OCNF:Starch (1:0.5 wt\%) gels. The longer hydrocarbon tail group of CTAB (C-16) compared to the C- 

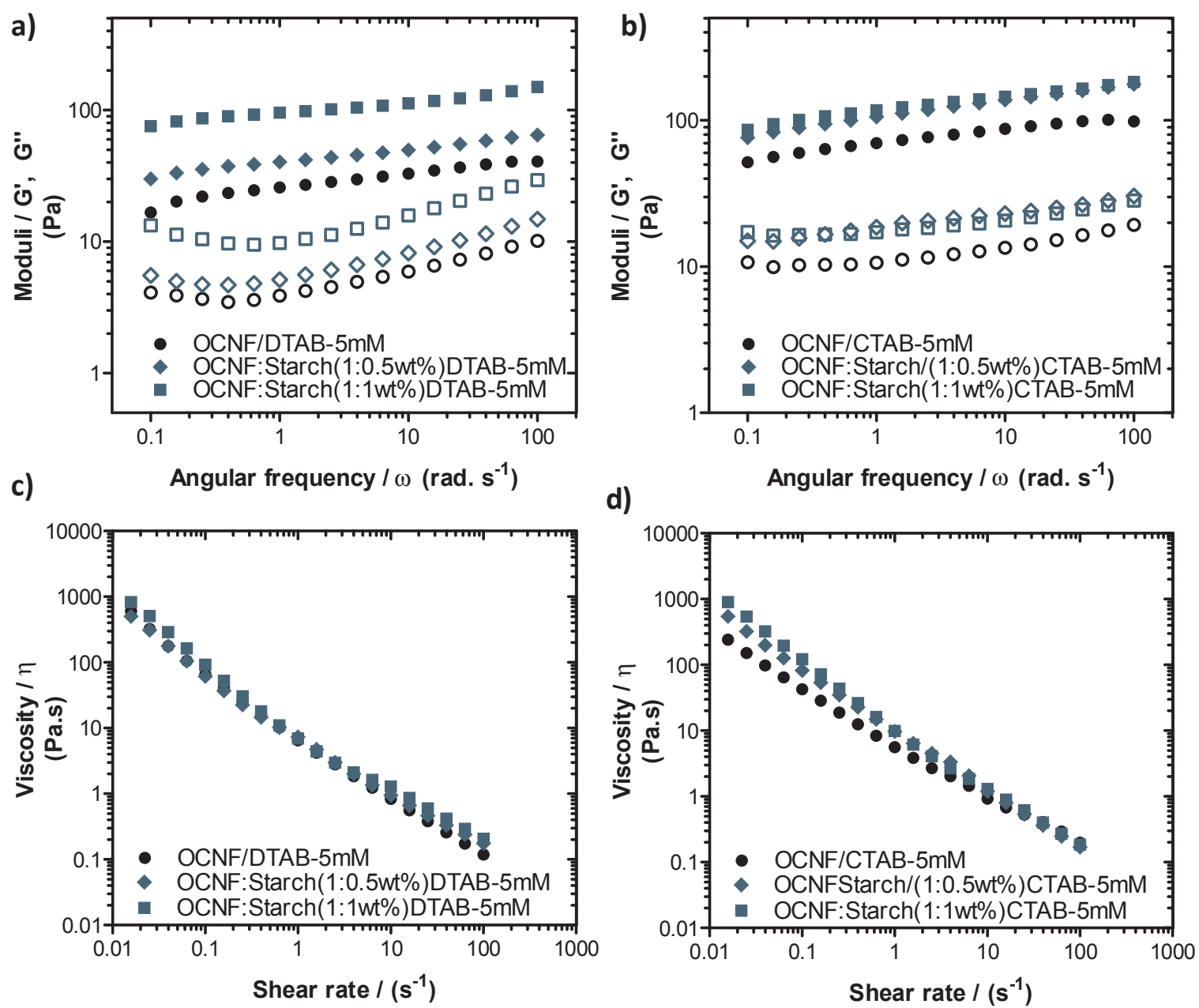

Fig. 5. a) Oscillatory frequency sweeps of OCNF:Starch/DTAB and b) OCNF:Starch/CTAB gels (closed and open symbols represent G' (storage modulus) and G" (loss modulus), respectively; c) shear flow curves of OCNF:Starch/DTAB and d) OCNF:Starch/CTAB gels.

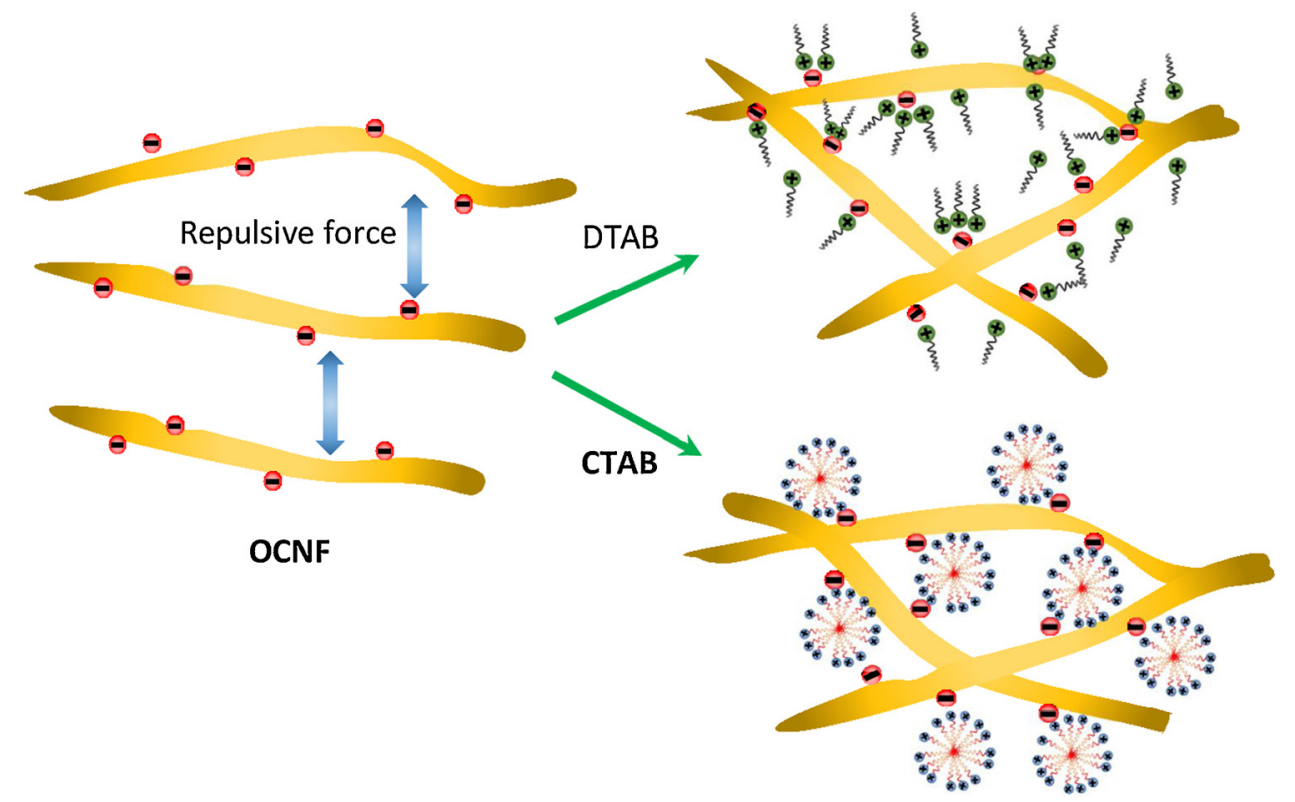

Fig. 6. Cartoon illustrations show the binding mechanism of DTAB and CTAB with the OCNF. Anionic cellulose fibrils show the electrostatic repulsive forces, while addition of cationic surfactants to the OCNF suspension reveals formation of hemimicelles (for DTAB at $5 \mathrm{mM}$ ) and micelles (for $\mathrm{CTAB}$ at $5 \mathrm{mM}$ ) inducing electrostatic attractive forces. Illustration not drawn to scale. 
12 tail group of DTAB means CTAB has a lower CMC (1.1 mM (Moulik et al., 1996; Tedeschi et al., 2003)) compared to that of DTAB ( $\sim 14.0 \mathrm{mM}$ (Moulik et al., 1996; Tedeschi et al., 2003)) in water. Thus at $5 \mathrm{mM} \mathrm{CTAB}$ is above its CMC and when it binds to the cellulose fibril surface, micelles may form, which can further bind to more of the anionic OCNF fibrils (see representative illustration in Fig. 6). DTAB however at $5 \mathrm{mM}$ is below its CMC, and binds to the OCNF as individual molecules or in the form of hemimicelles. The C12 chain will also in general provide a lower hydrophobic character to the surface than the C16 chain until all the anionic sites are occupied. Syneresis was also observed in OCNF:Starch (1:1 wt $\%$ )/CTAB gels after $24 \mathrm{~h}$ due to greater aggregation and subsequent sedimentation (ESI 5).

We also note that addition of surfactants (DTAB and CTAB) to the starch only solution (1 wt\%) did not reveal any remarkable improvement in their gel strength (i.e., no self-standing gels were formed, as can be seen in ESI 6) as was observed for OCNF/surfactants gels. OCNF has a negative charge $(-56 \pm 2 \mathrm{mV})$ due to the carboxylic acid whilst starch has a comparatively lower negative charge $(-13 \pm 2 \mathrm{mV})$ due to the hydroxyl groups ( $\zeta$-potential values are provided in the ESI 7 and 8). In addition, OCNF is a particle, expected to be stable as a dispersion in water only due to its strong electrostatic repulsion, while starch is a polymer with weak $\zeta$ - potential. Hence, the addition of surfactant counter ions is expected to have a different effect on starch and OCNF.

Addition of DTAB/CTAB to the OCNF:Starch system produced gels that also had shear-thinning properties, as presented in Fig. $5 \mathrm{c}-\mathrm{d}$. However, incorporation of DTAB in the OCNF:Starch gel system did not cause any significant change in the shear viscosity values compared to the OCNF/DTAB gels. Addition of CTAB revealed a slight increase in the shear viscosity $\eta$ of the gels compared to the OCNF/CTAB gels.

The interactions of starch with OCNF and surfactants were further investigated using amylose-iodine complex test. In this test, molecular iodine $\left(\mathrm{I}_{2}\right)$ in presence of potassium iodide (KI) forms polyiodide ions $\left(\mathrm{I}_{3}{ }^{-}\right.$or $\left.\mathrm{I}_{5}{ }^{-}\right)$which produce a linear polyiodide chain with an average length of $3.1 \AA$ (Zeta potential $-6( \pm 2) \mathrm{mV}$ ) (Saenger, 1984). The amylose helix has an outer diameter of $13.0 \AA$ and a central cavity of $5.0 \AA$ with a pitch of $8.0 \AA$, which provides enough space to accommodate the polyiodide chains (Saenger, 1984). Polyiodide inside the amylose cavity acts as a charge acceptor, while amylose acts as a donor, creating a charge transfer complex, leading to a blue colour of the sample (Liu, Fei, Maladen, Hamaker, \& Zhang, 2009; Putseys et al., 2010). Fig. 7a reveals the colour change phenomenon of the OCNF:Starch/surfactants gels in presence of iodine solution. OCNF gels (1 wt
\%) did not show any colour change in presence of iodine solution (see Fig. 7a). This is expected as, cellulose does not form a complex with the polyiodide ions as both are negatively charged and the cellulose does not have any hydrophobic cavities. The starch solution $(0.5 \mathrm{wt} \%)$ showed the expected colour change to blue after addition of iodine solution due to the formation of the amylose-iodine complex mentioned above. OCNF:Starch gels were also seen to change to a blue colour (see Fig. 7a); however, the intensity of the blue colour was lighter than that of control starch solution. It is likely that the starch polymer helices in solution are disrupted by the OCNF, possibly because it wraps around some parts of the fibrils.

OCNF:Starch (1:0.5 wt\%) gels in the presence of DTAB or CTAB $(5 \mathrm{mM})$ did not respond to the iodine solution (Fig. 7a), suggesting the electrostatic linking between the positively-charged surfactant and negatively-charged OCNF:Starch formed a more complex network, preventing iodide ions from entering the centre of the amylose helices. This complex network might be formed by electrostatically attaching the surfactant's head groups to the oppositely charged cellulose fibrils while anchoring their tail groups within the amylose helices.

Amylose-iodide complex tests of various combinations of the OCNF/ Starch/surfactant system in presence of the iodine solution were further characterised using a UV-vis spectrophotometer. A dilute solution (25x) of OCNF/Starch/surfactant was used for the spectroscopic analysis (Fig. 7b) and the absorption spectra are presented in Fig. 7c. The dilute solution of Starch and OCNF:Starch showed a broad absorption peak in the region of $550-750 \mathrm{~nm}$ with a peak maximum at $620 \mathrm{~nm}$ associated with the amylose-iodine complex, which correlates well with the literature values (Jiang et al., 2012; Knutson, 2000; Liu et al., 2009). A lower absorbance intensity in OCNF:Starch was observed compared to the starch solution, which is in good agreement with the comparatively lighter blue colour obtained for the OCNF:Starch, as can be seen in Fig. 7b. The purple colour obtained for the Starch-DTABiodine complex showed the absorbance peak shifted toward shorter wavelengths with a peak maximum at $570 \mathrm{~nm}$. Possibly interference of the cationic surfactant via interactions with the negatively-charged polyiodide ions (Naorem \& Devi, 2013) could explain the shift observed in the charge transfer complex. Such a shift of the amylose-iodine complex peak maximum to lower wavelength values may be due to the decrease in the length of the available helices due to incorporation of the DTAB tail, thus the number of polyiodide ions which can be accommodated inside the helical cavity also decreases (Bailey \& Whelan, 1961; Banks, Greenwood, \& Khan, 1971; Knutson, 2000). Here, the a)

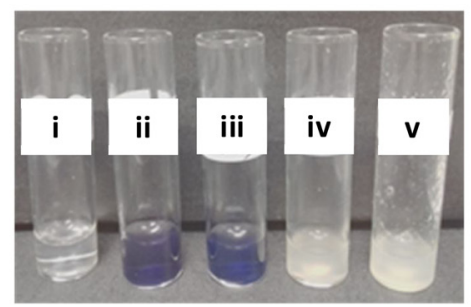

c)

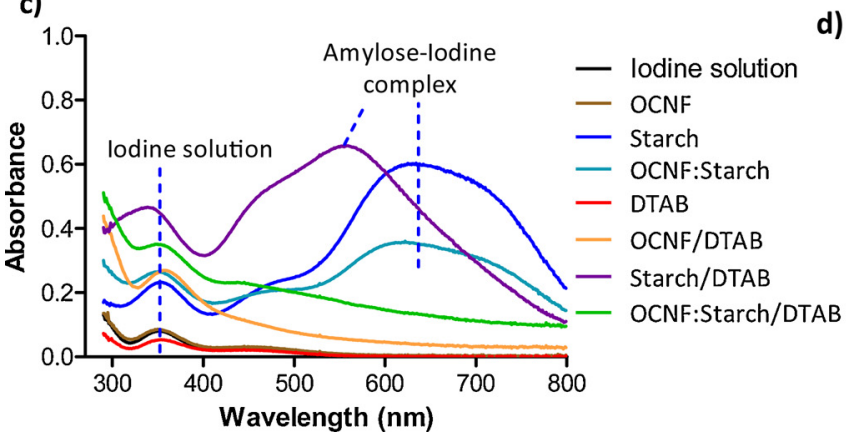

b)
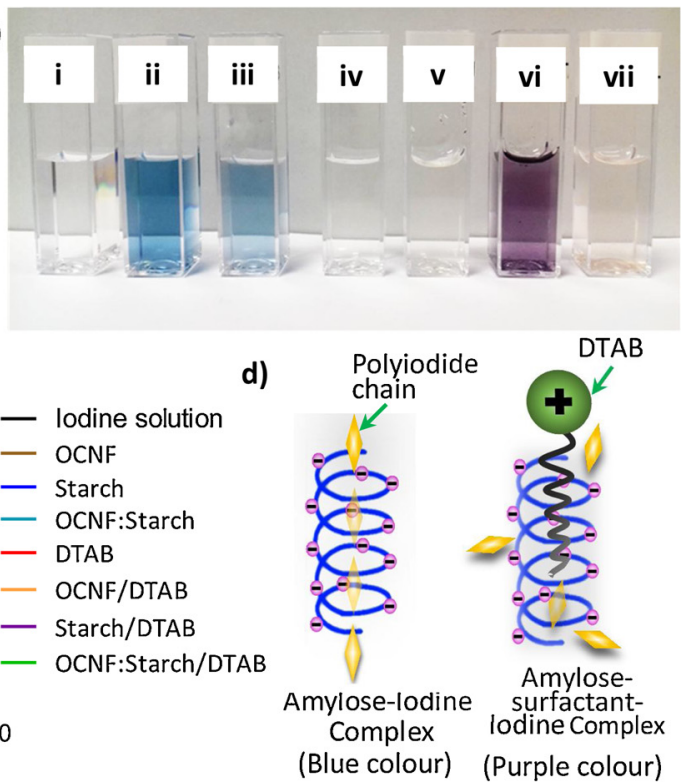

Fig. 7. a) Photographs showing the colour change phenomenon of the OCNF:Starch/ Surfactants gels with the addition of $\mathrm{I}_{2}$ solution (30 $\mu \mathrm{L}$ of $50 \%$ Lugol's solution) : (i) OCNF (1 wt\%), (ii) Starch $\quad(0.5 \mathrm{wt} \%), \quad$ (iii) OCNF:Starch (1:0.5 wt\%), (iv) OCNF:Starch $(1: 0.5 \mathrm{wt} \%) / \mathrm{DTAB} \quad(5 \mathrm{mM})$ and $(\mathrm{v})$ OCNF:Starch (1:0.5 wt $\%)-C T A B \quad(5 \mathrm{mM})$, b) dilute solution $(25 \mathrm{x})$ of various combination of OCNF/Starch/Surfactant system in presence of iodine solution used for UV-vis spectrophotometry analysis: (i) OCNF (1 \%), (ii) Starch (0.5\%), (iii) OCNF:Starch (1:0.5\%), (iv) DTAB (5 mM), (v) OCNF (1 \%)/DTAB (5 mM), (vi) Starch (0.5\%)-DTAB( $5 \mathrm{mM})$, (vii) OCNF:Starch (1:0.5 \%)/DTAB $(5 \mathrm{mM})$, c) UV-vis spectra of the corresponding dilute solutions with iodine, and d) illustration representing possible interactions in amylose/ DTAB/iodine solutions. 


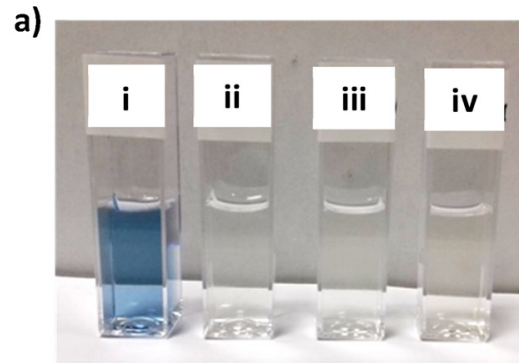

b)

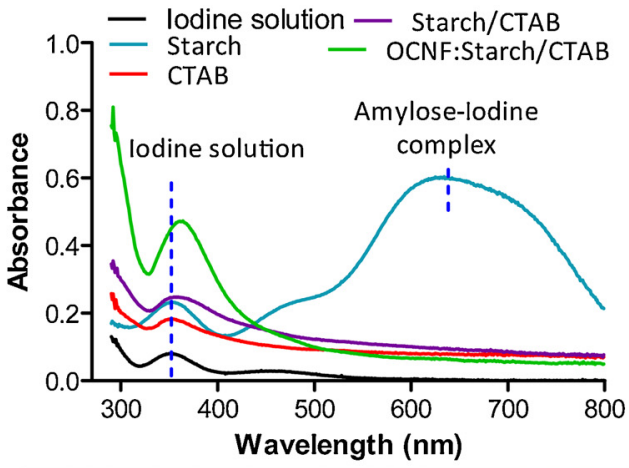

c)

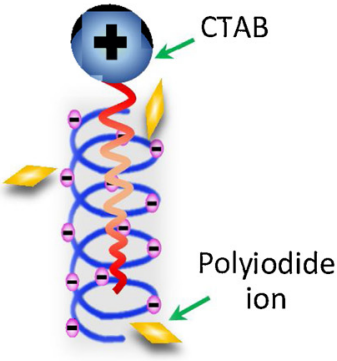

Amylose-CTAB-iodine complex
Fig. 8. a) Photographs showing the effect of addition of iodine solution to the dilute solution (25x) of (i) Starch (0.5\%) (ii) CTAB (5 mM) (iii) $\operatorname{Starch}(0.5 \%)-\mathrm{CTAB}(5 \mathrm{mM})$ (iv) OCNF:Starch(1:0.5 \%)/CTAB(5 mM), b) UV-vis spectra of the corresponding dilute solutions with iodine (i-iv), c) illustration representing probable interaction of amyloseCTAB-iodine complex, and d) photographs showing the colour change after addition of iodine solution ( $30 \mu \mathrm{L}$ of $50 \%$ Lugol's solution) into the diluted $(25 \mathrm{x})$ starch solution containing various concentrations of CTAB: i) $0 \mathrm{mM}$, ii) $0.25 \mathrm{mM}$, iii) $0.50 \mathrm{mM}$, iv) $1 \mathrm{mM} \mathrm{v}$ ) $2 \mathrm{mM}$, vi) $3 \mathrm{mM}$ and vii) $5 \mathrm{mM}$. interaction of the surfactant with the starch might be explained by anchoring of the hydrocarbon tail groups within the core of amylose helices, favouring hydrophobic interactions inside its cavity (Winter \& Sarko, 1974) and accommodating a lesser number of polyiodide ions, as illustrated in Fig. 7d. On the other hand, dilute solutions (25x) of not only OCNF, DTAB and OCNF/DTAB solution, but also the OCNF:Starch/ DTAB did not show the amylose-iodine complex associated absorption peak. In these solutions, only the iodine solution related absorbance peaks were seen at $320 \mathrm{~nm}$, giving clear indication that the cores of the starch helices were not available for iodine-starch complexation.

By comparison, incorporation of CTAB either in Starch or OCNF:Starch blends did not reveal any blue/purple colour. Fig. 8a presents the photographs of the diluted solution (25x) of Starch/CTAB mixtures. In addition, the absorbance peak (in the region of $550-750 \mathrm{~nm}$ ) associated with the amylose-iodine complex was absent for CTAB-Starch and OCNF:Starch/CTAB as can be seen in Fig. 8b. This may be due to the longer hydrocarbon tail group of CTAB (C-16), which is assumed to occupy the cavity of the amylose helix leaving no space for the iodide ion, as illustrated in Fig. 8c. On the contrary, when DTAB was used in starch solution, it is expected that the surfactant tail is too short to fill the entire helix so some polyiodide ions can still participate in the formation of amylose-iodine complex, as illustrated in Fig. 7d. Fig. 8d demonstrates that addition of sequentially more CTAB occupies an increasing number of binding sites in the starch helices until the system is saturated at $2 \mathrm{mM}$.

The iodine test experiment allows elucidation of the mechanisms of the interactions of the OCNF/Starch/surfactant hydrogels, where insertion of the surfactant tails into the starch helices is providing an extra source of binding in the starch-cellulose network to strengthen the gels. This correlates well with the improved rheological properties obtained for the surfactant-induced OCNF:Starch gels. The depth of the surfactant tail group anchored into the amylose helices is also believed to provide additional reinforcement in bridging the starch-cellulose network. Hence, higher $G^{\prime}$ value was obtained for the OCNF:Starch/ CTAB ( $5 \mathrm{mM})$ gels due to the longer tail group compared to the OCNF:Starch/DTAB ( $5 \mathrm{mM}$ ) gels. However, comparison of the rheological properties of the gels prepared at higher surfactant concentration $(10 \mathrm{mM})$ was avoided due to the gel instability, as syneresis was observed. Thus, the interaction among the cellulose fibrils, soluble starch and optimum concentration of surfactants $(5 \mathrm{mM})$ discussed in this paper may be useful in selecting these materials as a rheological modifier in formulations based products.

\section{Conclusions}

In this study, cationic surfactant (DTAB and CTAB) induced gelation of OCNF and soluble starch in water systems was investigated to determine their rheological behaviour. The increase of storage modulus of OCNF/surfactant hydrogels with increasing surfactant concentration demonstrates the progressive increase of the "stiffness" of the gels suggesting an electrostatic attraction between OCNF and DTAB/CTAB due to their oppositely charged moieties. Additionally, improved gel strength obtained with the incorporation of starch to the OCNF suspension was also suggested to form a network between the cellulose fibrils and amylose chains. The stiffness and viscosity of OCNF:Starch hydrogels were enhanced further by the addition of small amounts of cationic surfactants. The critical concentration of surfactant, as well as the solid content of gels, was suggested to form a stronger and stable surfactant-bridge between the cellulose nanofibrils and starch helices, which provides a clear understanding of these complex blend-surfactant networks that may have potential utility as rheological modifiers in formulated products.

\section{Acknowledgements}

The authors would like to thank EPSRC for funding this project (Grant EP/N033310/1). Mr Vincenzo Calabrese thanks the University of Bath for supporting his PhD. Mrs Ursula Potter (Microscopy and Analysis Suite, University of Bath) is thanked for her assistance in TEM measurements. Data supporting this work is freely accessible in the Bath research data archive system at DOI: https://doi.org/10.15125/ BATH-00683.

\section{Appendix A. Supplementary data}

Supplementary material related to this article can be found, in the online version, at doi:https://doi.org/10.1016/j.carbpol.2019.115816.

\section{References}

Azizi Samir, M. A. S., Alloin, F., \& Dufresne, A. (2005). Review of recent research into cellulosic whiskers, their properties and their application in nanocomposite field. Biomacromolecules, 6(2), 612-626. 
Bailey, J. M., \& Whelan, W. J. (1961). Physical properties of starch: I. Relationship between iodine stain and chain length. The Journal of Biological Chemistry, 236(4), 969-973.

Banks, W., Greenwood, C. T., \& Khan, K. M. (1971). The interaction of linear, amylose oligomers with iodine. Carbohydrate Research, 17(1), 25-33.

Brinatti, C., Huang, J., Berry, R. M., Tam, K. C., \& Loh, W. (2016). Structural and energetic studies on the interaction of cationic surfactants and cellulose nanocrystals. Langmuir, 32(3), 689-698.

Calabrese, V., da Silva, M. A., Schmitt, J., Muñoz-Garcia, J. C., Gabrielli, V., Scott, J. L., et al. (2018). Surfactant controlled zwitterionic cellulose nanofibril dispersions. Soft Matter, 14(38), 7793-7800.

Courtenay, J. C., Johns, M. A., Galembeck, F., Deneke, C., Lanzoni, E. M., Costa, C. A., et al. (2017). Surface modified cellulose scaffolds for tissue engineering. Cellulose, 24(1), 253-267.

Crawford, R. J., Edler, K. J., Lindhoud, S., Scott, J. L., \& Unali, G. (2012). Formation of shear thinning gels from partially oxidised cellulose nanofibrils. Green Chemistry, 14(2), 300-303.

da Silva, M. A., Calabrese, V., Schmitt, J., Celebi, D., Scott, J. L., \& Edler, K. J. (2018) Alcohol induced gelation of TEMPO-oxidized cellulose nanofibril dispersions. Soft Matter, 14(45), 9243-9249.

Dhar, N., Au, D., Berry, R. C., \& Tam, K. C. (2012). Interactions of nanocrystalline cellulose with an oppositely charged surfactant in aqueous medium. Colloids and Surfaces A: Physicochemical and Engineering Aspects, 415, 310-319.

Fall, A. B., Lindström, S. B., Sundman, O., Ödberg, L., \& Wågberg, L. (2011). Colloidal stability of aqueous nanofibrillated cellulose dispersions. Langmuir, 27(18), 11332-11338.

Fukuzumi, H., Tanaka, R., Saito, T., \& Isogai, A. (2014). Dispersion stability and aggregation behavior of TEMPO-oxidized cellulose nanofibrils in water as a function of salt addition. Cellulose, 21(3), 1553-1559.

Geng, L., Mittal, N., Zhan, C., Ansari, F., Sharma, P. R., Peng, X., et al. (2018). Understanding the mechanistic behavior of highly charged cellulose nanofibers in aqueous systems. Macromolecules, 51(4), 1498-1506.

Gong, J. P., Katsuyama, Y., Kurokawa, T., \& Osada, Y. (2003). Double-network hydrogels with extremely high mechanical strength. Advanced Materials, 15(14), 1155-1158.

Han, J., Zhou, C., Wu, Y., Liu, F., \& Wu, Q. (2013). Self-assembling behavior of cellulose nanoparticles during freeze-drying: Effect of suspension concentration, particle size, crystal structure, and surface charge. Biomacromolecules, 14(5), 1529-1540.

Haque, M. A., Kurokawa, T., \& Gong, J. P. (2012). Super tough double network hydrogels and their application as biomaterials. Polymer, 53(9), 1805-1822.

Hong, Y., Liu, G., \& Gu, Z. (2016). Recent advances of starch-based excipients used in extended-release tablets: A review. Drug Delivery, 23(1), 12-20.

Immel, S., \& Lichtenthaler, F. W. (2000). The hydrophobic topographies of amylose and its blue iodine complex. Starch - Stärke, 52(1), 1-8.

Ingverud, T., Larsson, E., Hemmer, G., Rojas, R., Malkoch, M., \& Carlmark, A. (2016). High water-content thermoresponsive hydrogels via electrostatic macrocrosslinking of cellulose nanofibrils. Journal of Polymer Science Part A: Polymer Chemistry, 54(21), 3415-3424.

Isogai, A., Saito, T., \& Fukuzumi, H. (2011). TEMPO-oxidized cellulose nanofibers. Nanoscale, 3(1), 71-85.

Jiang, T.-Y., Ci, Y.-P., Chou, W.-I., Lee, Y.-C., Sun, Y.-J., Chou, W.-Y., et al. (2012). Two unique ligand-binding clamps of rhizopus oryzae starch binding domain for helical structure disruption of amylose. PLoS One, 7(7), e41131.

Jin, S., Wang, Y., He, J., Yang, Y., Yu, X., \& Yue, G. (2013). Preparation and properties of a degradable interpenetrating polymer networks based on starch with water retention, amelioration of soil, and slow release of nitrogen and phosphorus fertilizer. Journal of Applied Polymer Science, 128(1), 407-415.

Johns, M. A., Bernardes, A., De Azevêdo, E. R., Guimarães, F. E. G., Lowe, J. P., Gale, E. M., et al. (2017). On the subtle tuneability of cellulose hydrogels: Implications for binding of biomolecules demonstrated for CBM 1. Journal of Materials Chemistry B, 5(21), 3879-3887.

Kavanagh, G. M., \& Ross-Murphy, S. B. (1998). Rheological characterisation of polymer gels. Progress in Polymer Science, 23(3), 533-562.

Knutson, C. A. (2000). Evaluation of variations in amylose-iodine absorbance spectra.
Carbohydrate Polymers, 42(1), 65-72.

Liu, J., Fei, L., Maladen, M., Hamaker, B. R., \& Zhang, G. (2009). Iodine binding property of a ternary complex consisting of starch, protein, and free fatty acids. Carbohydrate Polymers, 75(2), 351-355.

McNamee, C. E., Sato, Y., Wiege, B., Furikado, I., Marefati, A., Nylander, T., et al. (2018). Rice starch particle interactions at air/aqueous interfaces-Effect of particle hydrophobicity and solution ionic strength. Frontiers in Chemistry, 6, 139.

Miles, M. J., Morris, V. J., Orford, P. D., \& Ring, S. G. (1985). The roles of amylose and amylopectin in the gelation and retrogradation of starch. Carbohydrate Research 135(2), 271-281.

Morris, V. J. (1990). Starch gelation and retrogradation. Trends in Food Science \& Technology, 1, 2-6.

Moulik, S. P., Haque, M. E., Jana, P. K., \& Das, A. R. (1996). Micellar properties of cationic surfactants in pure and mixed states. The Journal of Physical Chemistry, 100(2), 701-708.

Murthy, P. S. K., Mohan, Y. M., Sreeramulu, J., \& Raju, K. M. (2006). Semi-IPNs of starch and poly(acrylamide-co-sodium methacrylate): Preparation, swelling and diffusion characteristics evaluation. Reactive \& Functional Polymers, 66(12), 1482-1493.

Naorem, H., \& Devi, S. D. (2013). Spectrophotometric determination of the formation constant of triiodide ions in aqueous-organic solvent or polymer mixed media both in absence and presence of a surfactant. Spectrochimica Acta Part A: Molecular and Biomolecular Spectroscopy, 101, 67-73.

Nordenström, M., Fall, A., Nyström, G., \& Wågberg, L. (2017). Formation of colloidal nanocellulose glasses and gels. Langmuir, 33(38), 9772-9780.

Notley, S. M. (2008). Effect of introduced charge in cellulose gels on surface interactions and the adsorption of highly charged cationic polyelectrolytes. Physical Chemistry Chemical Physics, 10(13), 1819-1825.

Prathapan, R., Thapa, R., Garnier, G., \& Tabor, R. F. (2016). Modulating the zeta potential of cellulose nanocrystals using salts and surfactants. Colloids and Surfaces A: Physicochemical and Engineering Aspects, 509, 11-18.

Putseys, J. A., Lamberts, L., \& Delcour, J. A. (2010). Amylose-inclusion complexes: Formation, identity and physico-chemical properties. Journal of Cereal Science, 51(3), $238-247$.

Quennouz, N., Hashmi, S. M., Choi, H. S., Kim, J. W., \& Osuji, C. O. (2016). Rheology of cellulose nanofibrils in the presence of surfactants. Soft Matter, 12(1), 157-164.

Sadasivam, S., \& Manickam, A. (1996). Biochemical methods. India: New Age International Publishers.

Saenger, W. (1984). The structure of the blue starch-iodine complex. Naturwissenschaften, $71(1), 31-36$.

Saha, D., \& Bhattacharya, S. (2010). Hydrocolloids as thickening and gelling agents in food: A critical review. Journal of Food Science and Technology, 47(6), 587-597.

Saito, T., Kimura, S., Nishiyama, Y., \& Isogai, A. (2007). Cellulose nanofibers prepared by TEMPO-mediated oxidation of native cellulose. Biomacromolecules, 8(8), 2485-2491.

Saito, T., Uematsu, T., Kimura, S., Enomae, T., \& Isogai, A. (2011). Self-aligned integration of native cellulose nanofibrils towards producing diverse bulk materials. Soft Matter, 7(19), 8804-8809.

Schmitt, J., Calabrese, V., da Silva, M. A., Lindhoud, S., Alfredsson, V., Scott, J. L., et al. (2018). TEMPO-oxidised cellulose nanofibrils; probing the mechanisms of gelation via small angle X-ray scattering. Physical Chemistry Chemical Physics, 20(23), 16012-16020.

Tardy, B. L., Yokota, S., Ago, M., Xiang, W., Kondo, T., Bordes, R., et al. (2017). Nanocellulose-surfactant interactions. Current Opinion in Colloid \& Interface Science, $29,57-67$.

Tedeschi, A. M., Franco, L., Ruzzi, M., Paduano, L., Corvaja, C., \& D’Errico, G. (2003) Micellar aggregation of alkyltrimethylammonium bromide surfactants studied by electron paramagnetic resonance of an anionic nitroxide. Journal of the Chemical Society Faraday Transactions, 5(19), 4204-4209.

Winter, W. T., \& Sarko, A. (1974). Crystal and molecular structure of V-anhydrous amylose. Biopolymers, 13(7), 1447-1460.

Yuliana, M., Huynh, L.-H., Ho, Q.-P., Truong, C.-T., \& Ju, Y.-H. (2012). Defatted cashew nut shell starch as renewable polymeric material: Isolation and characterization. Carbohydrate Polymers, 87(4), 2576-2581. 